\section{Ein paar Fragen zu HIN}

\author{
M. Romanens
}

In der Schweizerischen Ärztezeitung wird mit dem Segen der FMH eine Internetplattform für sämtliche Schweizer FMH-Titelträger gefordert und gefördert [1]. Die Vorteile dieses Unterfangens liegen auf der Hand:

1. Rascher Datenaustausch (Wissen, Kongresse, Termine, Überweisungen usw.);

2. Funktionierende Verschlüsselungssoftware (ASAS);

3. Vereinheitlichte Internetstrukturen für alle FMHTitelträger.

Die Gefahren wurden im besagten Artikel ebenfalls erwähnt, es sollen gerade bei der HIN gewisse Probleme optimal gelöst worden sein (z.B. Schutz vor Computerviren). Das ist sicher richtig. Nur, das Sicherheitsproblem im Internet ist weniger das Internet selbst als die Mitarbeiter hinter den Firewalls.

Folgende Fragen sollten beantwortet werden:

1. HIN ist eine Aktiengesellschaft, mehrheitlich im Besitz der FMH: wie lauten die Statuten dieser Gesellschaft? Wer sonst kontrolliert das HIN?

2. Wozu dient das HIN?

Das HIN ist ja zunächst einfach ein Server, welcher Informationen abspeichert. Welche Informationen sollen darauf jedoch erscheinen? Was ist der Zweck des HIN?

\section{Wer kontrolliert das HIN?}

Als Medium der Zukunft der FMH sollte das HIN durch die Mitglieder der FMH auf demokratische Art kontrollierbar sein. Es sollte eine offene, für alle FMH-Titelträger einsehbare und mit Statuten geregelte Struktur erhalten, wo z.B. festgelegt wird, was auf dem Server gespeichert werden darf und wer zu exakt welchen Informationen Zugang haben darf oder nicht. Es braucht eine klare Regelung für Chaträume und Foren.

Die Werbeeinnahmen und allgemeine Werbefragen müssen statutarisch geregelt werden, auch betreffend Handhabung von Mailinglisten usw. und
Fragen der Aufdringlichkeit von Werbung, Kompatibilität mit den Richtlinien der FMH für Werbeaktivitäten. Angesichts des vorgesehenen Grossprojekts müssen die Sicherheitsstandards innerhalb des HIN perfektioniert werden, es müssen externe Sicherheitsexperten für ein internes Sicherheitskonzept zugezogen werden. Das ist auch eine Vertrauensfrage.

4. Dürfen auch vertrauliche E-mails mittels ASAS via HIN-Server transferiert werden?

Der eidgenössische Datenschutzbeauftragte empfiehlt im Infoblatt Nr. 1/2000: "Verschlüsseln Sie alle Ihre vertraulichen Daten. Neben Ihren E-mails (PGP: www.pgpi.com, www.hushmail.com) können Sie die Daten auch auf Ihrer Festplatte verschlüsseln [...].» PGP heisst "pretty good privacy», eine Gratissoftware des Massachusetts Institute of Technology, der derzeitige Standard weltweit für die Verschlüsselung. Dank der dezentralen Anwendung dieses Systems, welches erst noch gratis und relativ einfach zu handhaben ist, ist das PGP-Netz nicht angreifbar. PGP wäre selbstverständlich auch innerhalb des HIN-Servers verwendbar.

Bevor vertrauliche Emails mittels ASAS transferiert werden dürfen, sollten die rechtlichen Aspekte eines solchen Transfers abgeklärt werden. Denkbar ist z.B., dass die E-mails auf dem HIN-Server unauffällig von Unbefugten entschlüsselt werden könnten.

Vielleicht wäre es besser, wenn die FMH das Know-how von PGP diffundiert, um den Transfer vertraulicher Daten via Internet den derzeitigen international gültigen Standards anzupassen.

Es ist sicher sinnvoll, möglichst rasch effiziente Kommunikationsstrukturen via Internet den interessierten Kolleginnen und Kollegen zur Verfügung zu stellen. Um die Schwelle für alle so niedrig wie möglich zu halten, werden im besagten Artikel sogar "plug and play"-Computer (gratis?) angeboten, so dass das Internet und HIN schon ab IQ 90 zugänglich wäre. Wir sollten es uns aber nicht zu einfach machen. Schliesslich wird der HIN-Server als Informationszentrum ein Machtinstrument ersten Ranges. Es ist höchste Zeit für eine detailliertere und kritische Betrachtung der HIN-Strukturen und Entscheidungsbäume durch die potentiellen HIN-Benutzer. $\mathrm{Zu}$ Fragen ist ferner, ob die heutige rechtliche Form des HIN als Aktiengesellschaft im Zuge der geplanten Umstrukturierungen und Plattformausweitung die richtige ist.
Korrespondenz:

Dr. med. Michel Romanens

Belchenstrasse 18

CH-4600 Olten

\section{Literatur}

1 Deschenaux F-X, von Below G. Health-Info-Net: Die Chance jetzt nutzen! Schweiz Ärztezeitung 2000;81(23):1215-6. 